\title{
Various Facets of Osteoarthritis Management of Osteoarthritis Are we getting it right?
}

\author{
S. S. Jha \\ Patron \& Founder President, Indian Orthopaedic Rheumatology Association, Lucknow, Uttar Pradesh, India
}

Corresponding Author:

Email: drssjha@gmail.com

\section{OA: No More Sequelae of Ageing}

$\mathrm{OA}$ is a common heterogenous disease with multiple etiologies but primarily is considered to be a degenerative joint disorder that presents with pain, joint swelling and limited mobility. Historically, considered to be a sequelae of ageing, resulting from focal 'wear and tear' of articular cartilage in response to mechanical damage. ${ }^{10}$ This perception needs to be drastically changed that $\mathrm{OA}$ is a condition of normal aging and also the fact the treatment options are limited.

Osteoarthritis, with increasing longevity / survival rates has substantial bearing on person's agility and acts of daily leaving (ADL). Driven by the present scenario 1.3 million knee replacements will have been performed in two years time in USA. By 2030, one fourth population of USA will have osteoarthritis with functional limitations of hip and knee, $35 \%$ of this will experience constraints in physical activity caused by this disease.

The current dogma is that both mechanics and genetics contribute to the development and progression of OA, depending on the anatomic location. OA is not a focal disease rather is a non-classical inflammatory disease of diarthrodial joints with presence of synoviocyte hypertrophy, pro-inflammatory cytokines and differentially affected sub-chondral bone compartments. The pro-inflammatory cytokines IL-1 $\beta$ and TNF are produced ultimately leading to loss of tensile strength. Cytokines IL-1 $\beta$ and TNF inturn induces the expression of other cytokines (IL-6 and IL8 ), chemokines (monocyte chemotactic protein 1 and granulocyte-macrophage colony-stimulating factor) and catabolic enzymes that we responsible for breakdown of cartilage and proteoglycans (matrix metalloproteinases (MMPs) and aggrecanases).

Under healthy conditions synoviocytes produce synovial fluid to nourish and lubricate articular cartilage. Thereby contributing to cartilage homeostasis. In OA, the synovium contributes to articular cartilage catabolism by-products of breakdown of cartilage extracellular matrix such as fibronectin and collagen fragments, induce inflammation in extant chondrocytes $^{6-7}$ and adjacent synovium. The activated macrophage-like synovial cells express TNF and IL-1 $\beta$, which inturn induce fibroblast-like synoviocytes to secrete other chemokines and cytokines.
Concommitently MMP-1, MMP-3, MMP-9 and MMP13 are expressed by fibroblast like synoviocytes and further contribute to cartilage degradation. CX43 is increased in the synovium and articular chondrocytes of patients with $\mathrm{OA}$, and is positively regulated by $\mathrm{IL}-1 \beta$ in both fibroblast-like synoviocytes and chondrocytes. Over-expression of CX43 in fibroblast-like synoviocsytes increases the expression of MMPs aggreecanases and pro-inflammatory cytokines through a mechanism dependent of nuclear factor $\mathrm{kB} .^{5}$ It is noteworthy that in rheumatoid arthritis also CX43 is observed in all tissues of the joint highlighting its involvement not only OA but in RA also.

\section{Obesity, Opiods and Imaging}

The approach to conservative management of osteoarthritis is customary prescription of NSAID/analgesic and or intra-articular glucocorticoid. On failure to achieve amelioration of symptoms and signs, joint replacement is usually recommended.

This is an inappropriate approach. The management plan has to be tailored to the individual's hardship and acknowledge their requirements in their set up. Ideally, pain and functional disabilities should form the central targets of management protocol. So, naturally a question arises: are we still getting the priorities right?

Plenty of guidelines have been developed for management of osteoarthritis but they do not correlate well with the required management of osteoarthritis. Hence, majority of patients do not get the right care. Health care cost also becomes an important factor. Now there is general awareness that glucosamine, paracetamol, opiods, viscosupplementation and arthroscopy-constitute palliative treatment, which frequently have no clinical benefit. They may be even harmful and not cost effective.

Efficacious evidence-based lifestyle behavior management strategies such as exercise and weight loss have never been fully emphasised. Inculcation of behavioral changes in the patient to perform exercise and undertake weight loss programme will initiate holistic approach at a minimal cost.

Yu et al. (2017) in their recent study has drawn attention regarding liberal use of opiods, which have substantial risk, as well. It should be reserved for 
patient not opting for total knee arthroplasty but should have a limited role with watchful eye on reports of adverse effects.

$\mathrm{Yu}$ et al. (2017) have also highlighted in their exhaustive study regarding abuse of role of radiography for diagnosis and staging of the disease and in management of osteoarthritis. Imaging invariably does not influence management protocol. Imaging features typically bear a poor correlation to symptoms.

Best possible evidence based management should form the basis of decision making with an eye on patient's safety, individual needs and goals. Monitoring of patient's response on regular basis is mandatory. Patient's knowledge of osteoarthritis and previous treatment, current level of pain and functional impairment, mood and sleep disturbance, presence of co-morbidities and patient's expectations of treatment are the important management strategies to be adopted by the clinicians. An early diagnosis of osteoarthritis enhances the opportunity to provide meaningful therapeutic benefit early in disease process.

\section{Recent Advances in Management}

Introduction of new therapeutic agents and introduction of evolution of sophisticated drug-delivery systems have been interesting advances in the past few years in the management of osteoarthritis. ${ }^{3}$ Consequences of long term use of conventional intraarticular glucocorticoids in the treatment of $\mathrm{OA}$ and evolution of a small-molecule inhibitor of the Wnt signaling pathway, sm04690 (Wnt pathway inhibitor) with possibilities of disease modifying OA, deserves attention for the management of OA. The Wnt signaling pathway stimulates the production of catabolic proteases that have been implicated in matrix degradation and modulates the differentiation of osteoblasts and chondrocytes. Intra-articular SM04690 might cause cartilage regeneration and provide protection from cartilage catabolism. ${ }^{2}$ A significant difference in joint space width is claimed to be observed. A larger phase two trial is expected soon which will fully examine the effects of SM04690. ${ }^{1}$

Clinical trials suggest declining analgesic effect of most widely recommended intra-articular glucocorticoid within 2-4 weeks. ${ }^{11}$ In an attempt to prolong the action of glucocorticoids, new delivery systems such as poly (PLGA:Poly Lactic-co-Glycolic Acid) microspheres have been introduced. An extended-release formulation of triamcelonone acetonide in PLGA microspheres, FX006 has been developed to maintain the concentration of this synthetic glucocorticoid in the joint for several months after a single dose. It claims to provide greater pain relief from 5 to 10 weeks. FDA approval was granted to FX006 for the management of knee OA. This approval represents a technological advance in providing a model for targeted delivery approach in treatment of OA.
Degree of joint space narrowing is positively associated with subsequent total knee arthroplasty within 15 years. For each $1 \%$ rate of tibial cartilage loss there is $20 \%$ increased risk of undergoing total knee arthrtoplasty within 4 years. ${ }^{1}$

\section{Stem Cell: Mesenchymal and Hematopoetic}

Role of mesenchymal stem cells (MSCs) in the repair of joint damage is too clearly understood. MSCs differ from bone marrow residing hematopoetic stem cells and are present in the joint in subchondral bone, cartilage, synovial fluid, synovium and adipose tissues. Migration of MSCs adjacent to the joint cavity becomes crucial for chondrogenesis ${ }^{4}$-has been suggested in experimental models during embryogenesis. Synovium derived MSCs is advocated to be the primary drivers of cartilage repair in adulthood-is also suggested in experimental models. Joint-resident MSCs with access to superficial cartilage become key cells in adult cartilage repair and represent important targets for manipulation in chondrogenic osteoarthritis (OA) especially in the context of biomechanical correction of joints in early disease. Joint resident MSCs are abundant in vivo and are likely to occupy multiple nichs in the joint, thus enabling single-stage therapeutic intervention in osteoarthritis. Contrary to this, native bone marrow-resident MSCs are being linked with a nerve growth factor (NGF), CD271. This opens a gateway of vital role of neutrophils in OA pathobiology but the implications needs exploration because any antiNGF therapy might worsen OA. Disease initiation and progression in pathogenesis of osteoarthritis is complex. In development of therapies for osteoarthritis especially for chondrogenic osteoarthritis, abundant literature is available emphasizing the role of culture expanded cellular therapies and scapholds. In chondrogenic OA, disease initiation and progression seems to be essentially dependent on the articular cartilage. Role of subchondral bone, including the osteochondral junction becomes important in the pathogenesis of OA. ${ }^{4}$ Role of native bone marrowresident stem cells, especially at sites of cartilage denudation needs to be further explored in advanced OA where such topographically localized cells can directly reach the joint cavity.

Spontaneous MSCs-mediated repair can happen in vivo has been realized. The mere fact that native MSCs are relatively abundant in the joint cavity, can be recruited to heal the cartilage defect. Remarkable structural repair of cartilage damage is demonstrated by total joint distraction ${ }^{8,13}$ procedures and osteotomies. ${ }^{9}$ Even topographic placing of MSCs at sites of injuries initiates the repair. These highlight, how intrinsic joint repair might be harmless. A window of opportunity is provided by removing the mechanical load and stopping the destructive forces on the damaged cartilage. It re-establishes joint haemostasis. 


\section{OA Phenotypes}

There is no generally accepted classification system for OA phenotypes, but in clinical practice the sub-group of varus aligned knee OA receives targeted treatment. Dell'Isola et al. (2016) in their aim to identify the various phenotypes proposed several phenotypes: chronic pain-associated OA with prominent central mechanism; inflammation-associated $\mathrm{OA}$, metabolic syndrome-associated $\mathrm{OA}$; associated with joint-localized bone and cartilage metabolism; mechanical load-associated OA; OA with minimal disease and low rate of progression. Disease phenotypes are meaningful, when they reflect differential treatment effects, prognosis or aetiology regardless of whether they are based on single factor or multiple factors. It can be finally concluded that patients with OA can be divided into subgroups of phenotypes on the basis of different dimension of disease: on aetiological phenotypes, structural/symptomatic presentation phenotypes, pain phenotypes, joint function related phenotypes, disability related phenotypes.

\section{Gene Therapy}

Intra-articular gene therapy was initially developed for overcoming the pharmacokinetic barriers in delivering biologics to the joints. It has great potential in the treatment of multiple diseases including OA (and RA also). After more than 25 years of development, arthritis gene therapy is finally entering in clinical practice. In 2017, the FDA approved three new gene therapeutics ${ }^{12}$ and the South Korean Ministry of Food \& Drug Safety has approved the first Arthritis Gene Therapy: invossa.

Upon successful gene transfer \& expression of the gene in the joint by residing cells, the therapeutic gene products are synthesized indigenously and continue to be synthesized for potentially extended period of time.

In vivo gene delivery to the joint by direct intraarticular injection is an alternative way to expedite the treatment. Adino-associated virus (AAV) has emerged as a popular vector for invivo delivery because the virus is safe, effective and less immunogenic. When injected into joints, recombinant AAV transduces synovial lining cells as well as chondrocytes throughout the thickness of the articular cartilage. This is a considerable advantage in $\mathrm{OA}$ in which chondrocyte dysfunction has a key role.

A faster approval is expected from FDA for these gene therapy new drugs. Invossa is only the fifth gene therapy product to be approved for anywhere in the world and the second for a non-lethal disease. Rapid future progress in genetic medicine for arthritis and other joint diseases is likely to be widely available by the middle of next decade.

\section{References}

1. Cicuttini, F. M. et al. Rate of cartilage loss at two years predicts subsequent total knee arthroplasty: a prospective study. Ann Rheum Dis 2004;63:1124-27.

2. Deshmukh, V. et al. A small-molecule inhibitor of the Wnt pathway (SM04690) as a potential disease modifying agent for the treatment of osteoarthritis of the knee. Osteoarthritis Cartilage 2018;26:18-27.

3. Flexion Therapeutics Inc. Zilretta (triamcinolone acetonide extended-release injectable suspension), for intra-articular use [label] Food and Drug

Administration

https://www.accessdata.fda.gov/drugsatfda_docs/label/2 017/208845s000lbl.pdf (2017).

4. Goldring, S. R. \& Goldring, M. B. Changes in the osteochondral unit during osteoarthritis: structure, function and cartilage-bone crosstalk. Nat Rev Rheumatol 2016;12:632-44.

5. Gupta, A. et al. Connexin 43 enhances the expression of osteoarthritis-associated genes in synovial fibroblasts in culture. BMC Musculoskelet Disord 2014;15:425.

6. Homandberg, G. A., Meyers, R. \& Williams, J. M. Intraarticular injection of fibronectin fragments causes severe depletion of cartilage proteoglycans in vivo. $J$ Rheumatol 1993;20:1378-82.

7. Homandberg, G. A. \& Hui, F. Association of proteoglycan degradation with catabolic cytokine and stromelysin release from cartilage cultured with fibronectin fragments. Arch Biochem Biophys 1996;334:325-31.

8. Intema, F. et al. Tissue structure modification in knee osteoarthritis by use of joint distraction: an open 1-year pilot study. Ann Rheum Dis 2011;70:1441-46.

9. Koshino, T., Wada, S., Ara, Y. \& Saito,

T. Regeneration of degenerated articular cartilage after high tibial valgus osteotomy for medial compartmental osteoarthritis of the knee. Knee 2003;10:229-36.

10. Malemud, C. J. Biologic basis of osteoarthritis: state of the evidence. Curr Opin Rheumatol 2015;27:289-94.

11. McAlindon, T. E. et al. OARSI guidelines for the nonsurgical management of knee osteoarthritis. Osteoarthritis Cartilage 2014;22:363-88.

12. US Food and Drug Administration. Approved cellular and gene therapy products. US Food and Drug Administration. FDAhttps://www.fda.gov/BiologicsBloodVaccines/Cellul arGeneTherapyProducts/ApprovedProducts/default.htm (2018).

13. Wiegant, K. et al. Sustained clinical and structural benefit after joint distraction in the treatment of severe knee osteoarthritis. Osteoarthritis Cartilage 2013;21:1660-67.

How to cite this article: S. S. Jha. Various Facets of Osteoarthritis Management of Osteoarthritis Are we getting it right? IP Int J Orthop Rheumatol 2018;4(2):40-2. 